\title{
Criptococosis cerebral en un paciente inmunocompetente (Presentación de un caso)
}

\section{Cryptococcal meningitis in immunocompetent patient \\ (Case presentation)}

\author{
Javier Lanchipa Picoaga $^{1 \mathrm{a}}$, Karina Moscoso Arana ${ }^{2 b}$ \\ ${ }^{1}$ Servicio de Medicina y Emergencia, Hospital Hipólito Unanue. Tacna, Perú. \\ ${ }^{2}$ Hospital Hipólito Unanue. Tacna, Perú. \\ ${ }^{a}$ Médico Internista, Asistente SME, Docente, Magister en Docencia Universitaria. \\ ${ }^{\mathrm{b}}$ Médico residente de Medicina Interna.
}

\begin{abstract}
Resumen
El Cryptococcus neoformans es un hongo levaduriforme encapsulado. Es el agente causal de la criptococosis, infección micótica profunda, la cual puede presentarse con un curso subagudo o crónico, generalmente como una infección oportunista. La meningitis criptocócica presenta características clínicas distintivas. La mayoría de las veces se adquiere fuera del hospital y se desarrolla subagudamente. Si no se trata oportunamente puede ser fatal en algunas semanas. Por tales razones se decidió la publicación del caso de un paciente masculino, de 58 años de edad, sin antecedentes ni evidencias de alteraciones de inmunidad, quien ingresó al hospital Hipólito Unanue de Tacna, con cuadro caracterizado por cefalea, convulsión focal, náuseas y vómitos explosivos. Al interrogatorio se recogió el antecedente del paciente de sufrir cefalea sin causa aparente desde hacía un tiempo atrás, la cual cedía con analgésicos. Se hospitalizó para la realización de estudios complementarios, en los que se concluyó el diagnóstico de criptococosis meníngea.
\end{abstract}

Palabras clave: Criptococosis cerebral, paciente inmunocompetente, infección cerebral.

\begin{abstract}
Cryptococcus neoformans is a yeast-capped fungus; it is the causal agent of cryptococcosis, deep fungal infection, which can present with a subacute or chronic course, usually as an opportunistic infection. Cryptococcal meningitis has distinctive clinical characteristic. In most cases it is acquired out of the hospital and develops sub acutely. If it is not treated promptly it can be fatal in a few weeks. For these reasons, it was decided to publish the case of a 58-year-old male patient, with no history or evidence of immune disorders, who was admitted to the Hipolito Unánue Hospital in Tacna, with a condition characterized by headache, focal seizure, nausea and explosive vomiting. On questioning, there was information of a personal antecedent of headaches without apparent cause for some time ago, that yield with pain relievers. He was hospitalized for complementary studies which concluded the diagnosis of cryptococcal meningitis.
\end{abstract}

Keywords: Cryptococcal meningitis, immunocompetent patient, brain infection.

\section{Introducción}

La criptococosis es una infección micótica de distribución mundial, producida principalmente por el complejo Cryptococcus neoformans / Cryptococcus gattii ( $C$. neoformans/C. gattii), que se encuentran ampliamente distribuidos en la naturaleza. La $C$. neoformans afecta principalmente a personas inmunocomprometidas, mientras que la $C$. gattii lo hace en pacientes inmunocompetentes expuestos al nicho ecológico del hongo (1).

Por lo general; la puerta de entrada es por vía respiratoria, por la inhalación de excremento de aves, principalmente palomas; y posteriormente se disemina a otras regiones del cuerpo, generalmente el sistema nervioso central (2).

En el cerebro provoca conglomerados de criptococos en zonas perivasculares de la sustancia gris cortical, ganglios basales y, en menor grado, en otros sitios del sistema nervioso central, es esta la localización en más del 75\% de los casos (3).

En las personas inmunocompetentes, por lo general, se manifiesta como infección pulmonar primaria; pero en las personas inmunodeprimidas, las manifestaciones iniciales, a menudo, se deben a la propagación hematógena a las meninges con meningitis subaguda o crónica. Otras localizaciones de la infección diseminada 
son los riñones, próstata, huesos y piel; en esta última, en forma de pústulas, pápulas, placas, úlceras o masas subcutáneas. La meningitis no tratada ocasiona la muerte en el término de semanas o meses. En pocos casos se describen infecciones cutáneas primarias (4).

El Cryptococcus neoformans puede afectar a individuos inmunocompetentes, a quienes causa infecciones localizadas clínicas y subclínicas, y los factores de riesgo más importantes identificados son: el uso de corticosteroides, los procesos linfoproliferativos y los trasplantes de órganos. La criptococosis es una entidad considerada emergente, dado la incidencia creciente en el mundo entero. No obstante, es una enfermedad poco común en pacientes sin inmunodepresión, aunque se han reportado varios casos en pacientes inmunocompetentes (4).

Se ha descrito una incidencia del $10 \%$ en pacientes expuestos al excremento de paloma y que son inmunocompetentes; a diferencia del $60 \%$ que se describe en pacientes inmunosuprimidos o con algún factor de riesgo. Se ha reportado que en pacientes inmunocompetentes existe una relación hombre: mujer de 2:1, mientras que en pacientes inmunocomprometidos hay una relación de hasta 11:1 (3).

Las manifestaciones clínicas de la criptococosis meníngea son similares a un cuadro de meningoencefalitis, donde los síntomas que predominan son la cefalea intensa, las alteraciones neurológicas y datos de inflamación encefálica (2).

El diagnóstico se hace por medio de la tinción del líquido cefalorraquídeo (LCR) con tinta china, donde se pueden observar las levaduras encapsuladas. En el LCR se puede encontrar líquido de aspecto transparente, disminución de glucosa o glucosa normal, elevación de proteínas o proteínas normales, celularidad aumentada con predominio de linfocitos (pleocitosis linfocitaria). Se encuentran alteraciones en la glucosa y en las proteínas en el $70 \%$ de los casos. La confirmación se hace por medio de un cultivo en agar Sabouraud (2).

Por ser poco común, y para demostrar la importancia de un diagnóstico precoz, se decidió la presentación de este caso.

\section{Caso clínico}

Paciente masculino de 58 años de edad, natural y procedente de Tacna, casado; inicia el padecimiento hace 8 meses aproximadamente, previos a su ingreso por cefalea holocraneana esporádica, que cedían al inicio con analgésicos. Dos meses, aproximadamente, antes de su ingreso, la cefalea se hace más frecuente, con moderada intensidad, con exacerbación a la luz y disminución del dolor con el reposo y analgésicos. Hijo del paciente refiere que su padre también presentó un episodio de movimiento involuntario de extremidad superior derecha, por aproximadamente 7 minutos, con posterior estado de somnolencia, motivo por el cual el paciente es visto por especialista particular, quien le indica, además de analgésicos, un medicamento antivertiginoso.

A 15 días antes de su ingreso, la cefalea holocraneana se exacerba, siendo calmada levemente con medicación indicada; sin embargo, al presentar vómitos explosivos, sumado a un mayor compromiso general, es llevado por sus familiares a Emergencias de la institución.

Se le realiza tomografía cerebral (Fig. 1) y una punción lumbar, donde se reportaron resultados patológicos que correspondían a una posible infección fúngica (Tabla 1). Asimismo, se le realizó una tinción del LCR con tinta china, donde se detectó la presencia de estructuras compatibles a Criptococcus $2+$, por lo que se le realiza un cultivo que resultó positivo a $C$. neoformans.

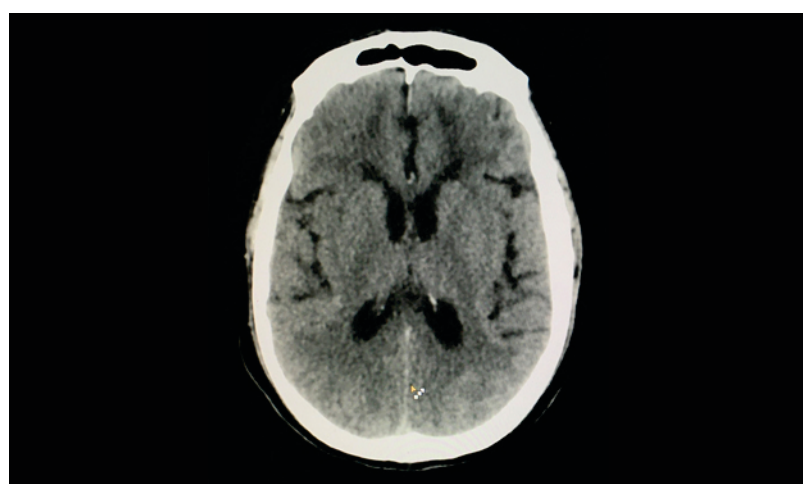

Figura 1. TAC de cráneo sin administración de contraste. Imagen con corte transversal a nivel de senos frontales. No se encuentran aparentemente alteraciones a nivel de cráneo ni en tejido cerebral.

Tabla 1: Resultados del análisis de LCR

\begin{tabular}{ll}
\hline Color & Incoloro \\
Aspecto & Transparente \\
Coágulo & No hay \\
Glucosa & $20.0 \mathrm{mg} / \mathrm{dl}$ \\
Proteínas & $89.91 \mathrm{mg} / \mathrm{dl}$ \\
Recuento Celular & 2 cél. x mm \\
\hline
\end{tabular}

Fuente: Historia clínica.

Al no contar con anfotericina B en la institución, se inicia tratamiento con fluconazol, con el cual se comienza a ver una evolución lentamente favorable.

Durante la hospitalización también se le realizan estudios serológicos, buscando inmunosupresión, los cuales resultan negativos. Se averigua que en su anterior residencia el techo era de calamina y palomas solían guarecerse en espacios libres.

A la segunda semana de tratamiento presenta nuevo cuadro de cefalea intensa, realizándose una segunda punción lumbar, tras lo cual el paciente presenta 
disminución de la cefalea. Posteriormente presenta cuadro de insuficiencia respiratoria; por lo que se le realizan varios exámenes, incluyendo tomografía torácica, más cultivos. Se le diagnóstica neumonía intrahospitalaria, por lo cual recibe tratamiento antibiótico.

A los 33 días de haber iniciado el tratamiento con fluconazol y haber finalizado el tratamiento antibiótico con una notoria mejoría, el paciente es dado de alta.

\section{Discusión}

La criptococosis es una infección que va en aumento desde los años 80, debido principalmente a la epidemia VIH y a las condiciones asociadas a la inmunosupresión. La criptococosis meníngea en pacientes inmunocompetentes es extremadamente infrecuente en comparación con la presentación en pacientes inmunocomprometidos, ocupando un $10 \%$ de los casos. El agente causal más frecuente es $C$. neoformans. Su vía de entrada es respiratoria, la levadura se infiltra hacia el torrente sanguíneo y se disemina a los demás órganos, donde va a infectar principalmente al SNC. En pacientes inmunocompetentes la respuesta celular llega a contener ciertas cargas del hongo, mientras que en pacientes inmunosuprimidos las cargas del hongo en el suero son mayores, en especial en aquellos con un recuento de CD4 $<100 / \mu 1$.

La manifestación clínica habitual es la infección del SNC, en general con un cuadro de meningoencefalitis subaguda o crónica. Los síntomas orientadores son la fiebre y la cefalea; menos frecuente los síntomas meníngeos, de focalización o alteración de conciencia. El segundo órgano afectado es el pulmón; le siguen en frecuencia el riñón y la próstata. El $C$. neoformans puede afectar a individuos inmunocompetentes, a los que causa infecciones localizadas, clínicas o subclínicas o de forma más frecuente a inmunosuprimidos, en los que la tendencia natural es la diseminación.

El diagnóstico temprano y oportuno es muy importante; ya que, el retraso del mismo y también el inicio del tratamiento puede condicionar la aparición de secuelas neurológicas, o llegar inclusive a la muerte. Se debe buscar el antecedente de exposición al excremento de aves, u ocupaciones que impliquen el cuidado o uso de las mismas mediante la historia clínica, para posteriormente continuar con el examen físico. Es importante la realización inmediata de una punción lumbar donde se detecte el hongo característico de celularidad aumentada con predominio de monocitos, proteínas elevadas o normales y glucosa baja o normal. Al LCR por sospecha, se debe hacer posteriormente la demostración de la cápsula del hongo por medio del uso de tinta china, que tiene una sensibilidad del 70 a $90 \%$ en pacientes VIH, y
$50 \%$ en pacientes sanos para la detección del hongo. Finalmente, la detección se hace con un cultivo hecho en agar Saboraud, el cual es considerado prueba de elección. También se usa la prueba de detección del antígeno capsular, ya que tiene una sensibilidad de más o menos $90 \%$ en pacientes sanos. Existen otras pruebas alternativas, como la detección mediante inmunoanálisis o las pruebas de aglutinación que se pueden hacer en látex. Por otro lado, el uso de TAC o RM es fundamental para identificar alguna alteración o secuela, como son la presencia de abscesos en el caso de infiltración a SNC, o nódulos en casos de enfermedad pulmonar.

Actualmente, el tratamiento de primera línea en pacientes inmunocompetentes es un esquema con anfotericina B, desoxicolato con flucitosina durante, al menos, 4 semanas, como terapia de inducción, seguido de terapia de consolidación y mantenimiento con fluconazol, durante 6 a 12 semanas. La anfotericina B tiene innumerables reacciones adversas, por lo que se recomienda precauciones antes de su administración; dada su nefrotoxicidad, es necesaria la vigilancia de la función renal. Como medicamento alternativo estaría el fluconazol, que es un tiazólico diferente a los otros imidazólicos en el plano bioquímico y farmacológico, ya que es hidrosoluble, se difunde a través de las meninges y se elimina en forma activa. Sus resultados pueden compararse a aquellos obtenidos con la anfotericina B. Se administra por vía oral o endovenosa a la dosis de 50 a 400 $\mathrm{mg} / \mathrm{kg}$ /día en dosis única (máximo $800 \mathrm{mg}$ ). Entre sus efectos indeseables están los gastrointestinales, cefalea, rash cutáneo y el aumento de transaminasas, pero es rara la nefrotoxicidad grave.

\section{Conclusiones}

La meningitis criptocócica es una enfermedad oportunista y rara, y siempre debe considerarse como diagnóstico diferencial en pacientes con síntomas de meningitis. El diagnóstico clínico es difícil de establecer por el inicio subagudo de los síntomas y la manifestación poco específica. La única forma de llegar al diagnóstico es por medio de una punción lumbar, y la tinta china la herramienta que dará el diagnóstico definitivo, como sucedió en nuestro caso clínico.

El tratamiento aceptado de la criptococosis meníngea es con anfotericina con fluocitosina; sin embargo, este último medicamento es muy costoso y difícil de conseguir en nuestro medio. El hospital no contaba con anfotericina. El paciente de este reporte se trató con fluconazol a dosis altas.

Es importante prevenirla en pacientes inmunocompetentes, ya que se ha asociado con la muerte. Es vital hacer hincapié en el cuidado al manejar el excremento de las aves y evitar exponerse a ellas. 


\section{Referencias bibliográficas}

1. Rodríguez MTP. Chronic neurocryptococcosis in an immunocompetent patient. 2014; 6 . D i s p o n i b 1 e e $\mathrm{n}$ : http://scielo.sld.cu/pdf/rpr/v18n6/rpr20614.pdf

2. Galnares-Olalde JA, Loza-Jalil S, Gómez-Peña F, Muñoz-Abraham O, Pavía-Aubry V, de LunaGallardo D. Criptococosis meníngea en un paciente inmunocompetente: reporte de un caso y revisión de la literatura. Revista Médica Del Hospital General De México [Internet]. julio de 2014 [citado 7 de junio de 2020];77(3):137-41. D i s p o n i b l e e n : https://linkinghub.elsevier.com/retrieve/pii/S01 85106314000109
3. Martínez I, Moronta A, Nordelo Y, Borrego M, Álvarez S. Criptococosis cerebral en paciente inmunocompetente. Presentación de caso y revisión de la literatura [Internet]. 2013. D i s p o n i b l e

e $n$ : https://www.medigraphic.com/pdfs/mediciego/ mdc-2013/mdcs132w.pdf

4. Rodríguez IR, Cruz MOU, Sánchez MP. Meningoencefalitis por Cryptococcus. Presentación de un caso Meningoencephalitis by Cryptococcus. Case presentation. 2016;5. D i s p o n i b l e e n : http://scielo.sld.cu/pdf/ms/v14n6/ms16614.pdf
Correspondencia:

javlan33@hotmail.com

karo1169@hotmail.com
Fecha de recepción: 11 de mayo del 2020

Fecha de aceptación: 12 de junio del 2020 